\title{
Ag85A/ESAT-6 chimeric DNA vaccine induces an adverse response in tuberculosis-infected mice
}

\author{
YAN LIANG, XUEJUANG BAI, JUNXIAN ZHANG, JINGYING SONG, \\ YOURONG YANG, QI YU, NING LI and XUEQIONG WU
}

\begin{abstract}
Army Tuberculosis Prevention and Control Key Laboratory, Beijing Key Laboratory of New Techniques of Tuberculosis Diagnosis and Treatment, Institute of Tuberculosis Research, The 309th Hospital of Chinese PLA, Beijing 100091, P.R. China
\end{abstract}

Received May 21, 2015; Accepted March 24, 2016

DOI: $10.3892 / \mathrm{mmr} .2016 .5364$

\begin{abstract}
The Mycobacterium tuberculosis (M. tb) antigens encoded by the $6 \mathrm{kDa}$ early secretory antigenic target (esat-6) and antigen 85A (ag85a) genes are known to exert protective effects against tuberculosis in animal models. In addition, these antigens represent vaccine components that were tested in early human clinical trials. In the present study, a chimeric DNA vaccine was constructed that contained two copies of the esat- 6 gene inserted into the $a g 85 a$ gene from $M . t b$. BALB/c mice were treated with this chimeric vaccine following infection with either $M . t b \mathrm{H} 37 \mathrm{Rv}$ or a clinical multi-drug-resistant tuberculosis isolate. Treatment of both groups of mice with the chimeric vaccine resulted in accelerated mortality. These findings are in contrast with previous results, which indicated that DNA vaccines expressing the individual antigens were either beneficial or at least not harmful. The results of the present study suggested that the ESAT-6 antigen is not suitable for inclusion in therapeutic vaccines.
\end{abstract}

\section{Introduction}

Tuberculosis (TB) is a leading cause of morbidity and mortality worldwide, with 8.7 million new cases and 1.4 million cases of mortality attributed to TB in 2011 (1). Multi-drug-resistant TB (MDR-TB) has emerged as a novel challenge, particularly in developing countries. Approximately $60 \%$ of newly occurring MDR-TB cases occur in Southeast Asia and the Western

Correspondence to: Professor Xueqiong Wu, Army Tuberculosis Prevention and Control Key Laboratory, Beijing Key Laboratory of New Techniques of Tuberculosis Diagnosis and Treatment, Institute of Tuberculosis Research, The 309th Hospital of Chinese PLA, 17 Heishanhu Jia, Haidian, Beijing 100091, P.R. China E-mail:wu-xueqiong@263.net

Abbreviations: MDR-TB, multi-drug-resistant tuberculosis; TB, tuberculosis; M. tb, Mycobacterium tuberculosis; Th, T helper; $\mathrm{CFU}$, colony-forming units; RFP, rifampin; PZA, pyrazinamide

Key words: Ag85A/ESAT-6, DNA vaccine, hypersensitivity reaction, Mycobacterium tuberculosis
Pacific region (2). The increased prevalence of MDR-TB is predominantly associated with nonstandard chemotherapy and a lack of funding to support the treatment of MDR-TB with second-line anti-TB drugs (3). Another contributory factor is the long duration of chemotherapy, which may induce functional impairment of the liver and kidneys, consequently leading to premature cessation of treatment. China has the second highest prevalence of $\mathrm{TB}$, according to the fifth national epidemiological sampling survey of TB in China in 2010 (4). The incidence of active pulmonary TB was 459 per 100,000 inhabitants in China, and the incidence of smear-positive or culture-positive pulmonary TB was 66 or 119 per 100,000 inhabitants, respectively (4). TB control presents a major challenge in China, due to the high incidence of MDR-TB and the lack of funding for treatment with second-line anti-TB drugs. The treatment of patients with MDR-TB or serious liver injury is complex; therefore, a novel approach is required and therapeutic vaccination may be helpful. Previous studies have demonstrated that DNA vaccines exert profound therapeutic effects in mice with TB $(5,6)$. In addition, immunotherapy with plasmid DNA encoding mycobacterial antigens combined with conventional chemotherapy presents a more rapidly effective form of treatment for TB reactivation and reinfection $(7,8)$.

DNA vaccination has been pursued for the treatment of tuberculosis due to its ability to enhance cellular immune responses, including $\mathrm{T}$ helper (Th) type 1 and cytotoxic $\mathrm{T}$ lymphocyte immune responses, which are essential for the control of TB $(9,10)$. Lowrie et al (11) demonstrated that DNA vaccines, initially designed to prevent infection, may also have a pronounced therapeutic action. In animal models, immunotherapy with plasmid DNA is a valuable adjunct to anti-TB chemotherapy, which has been shown to shorten the duration and improve the treatment of latent TB infection (12). Heat shock protein 65 DNA is able to switch the immune response from one that is relatively inefficient and produces bacterial stasis, to one that kills bacteria in heavily infected mice (6). Ha et al (5) demonstrated that immunotherapy using a plasmid DNA vaccine encoding mycobacterial antigen 85A (Ag85A), combined with conventional chemotherapy, was highly effective for the prevention of Mycobacterium tuberculosis (M.tb) reactivation and reinfection in mice. Another strong T-cell antigen of $M . t b$ is $6 \mathrm{kDa}$ early secretory antigenic target (ESAT-6), which is absent from the current vaccine Bacillus 
Calmette-Guérin (BCG) (13), and is considered an attractive candidate for the development of novel TB vaccines (14). ESAT-6 is strongly recognized during the early phase of $M$. $t b$ infection, as well as in patients with active TB, and is a potent inducer of long-lasting memory immunity against TB (14,15). Both Ag85A and ESAT-6 represent components of TB vaccines that were tested in early clinical trials $(16,17)$.

To date, ESAT- 6 has been used to construct several types of TB vaccine, including recombinant BCG, DNA vaccines and subunit vaccines. Lowrie et al (11) reported that bacterial numbers in the lungs and spleen of mice were decreased, and T-cell profiles were altered towards a Th1-type dominant response following treatment with an ESAT-6 DNA vaccine, thus indicating that an ESAT-6 DNA vaccine may exert beneficial effects in mice with TB. Since the immunotherapeutic effects of single-antigen Ag85A DNA or ESAT-6 DNA vaccines are limited, the use of a DNA plasmid that expresses both as a chimeric protein is an attractive vaccine strategy (18). Chimeric DNA vaccines may exert stronger immunotherapeutic effects and reduce vaccine costs, as compared with a mixture of two vaccines. Furthermore, a fusion protein of the closely related Ag85B and ESAT-6 has been shown to generate good protective immunity in macaques (16). Accordingly, the present study constructed an Ag85A/ESAT-6 chimeric DNA vaccine, which consisted of one ag85a gene into which two copies of the esat- 6 gene were inserted (19). Our previous study demonstrated that the immunotherapeutic effects of an Ag85A/ESAT-6 chimeric DNA vaccine containing one copy of the esat- 6 gene were reduced compared with those of the Ag85A DNA vaccine in MDR-TB infected mice (20). The present study extended those observations, and demonstrated that immunotherapy with a chimeric DNA vaccine containing two copies of the esat- 6 gene unexpectedly enhanced mortality of mice infected with either MDR-TB or drug-sensitive M.tb.

\section{Materials and methods}

Plasmid DNA. Plasmid vector pVAX1 DNA, Ag85A DNA, and Ag85A/ESAT-6 chimeric DNA were purified by Shanghai H\&G Biotechnology Company (Shanghai, China) using the EndoFree plasmid purification kit (Qiagen, Hilden, Germany).

Mice. A total of 160 pathogen-free female BALB/c mice (age, 6-8 weeks) were purchased from the Academy of Military Medical Sciences (Beijing, China). The mice were maintained in a level-2 negative pressure biosafety biohazard laboratory at the 309th Hospital of Chinese PLA (Beijing, China) with $-40 \mathrm{~Pa}$ room pressure, $-10 \mathrm{~Pa}$ cage pressure, $22 \pm 2^{\circ} \mathrm{C}$ temperature, $55 \pm 5 \%$ relative humidity and a $12 \mathrm{~h} \mathrm{light/dark} \mathrm{cycle.}$ The mice were fed a sterile commercial mouse diet (Beijing Keaoxieli Feed Co., Ltd., Beijing, China). The study was approved by the Ethics Committee of the 309th Hospital of the Chinese PLA (Beijing, China).

M. $t b$ strains. M. $t b$ standard strain $\mathrm{H} 37 \mathrm{Rv}$ and the clinical MDR-TB HB361 strain were used to infect the mice. The M. $t b \mathrm{H} 37 \mathrm{Rv}$ strain was provided by National Academy for the Control of Pharmaceutical and Biological Products (Beijing, China). The HB361 was a clincal strain isolated from a TB patient sputum sample at the Tuberculosis Department,
Thorax Disease Hospital (Shijiazhuang, China). The status was confirmed by conventional species identification, and conventional drug susceptibility testing using the absolute concentration method on Lowenstein-Jensen medium, in line with the Chinese Laboratory Science Procedure of Diagnostic Bacteriology in tuberculosis (21). The HB361 strain was resistant to rifampin (RFP), isoniazid and streptomycin, but was sensitive to pyrazinamide (PZA).

Generation and treatment of a mouse model of $T B$. In the first experiment, in order to evaluate the adjunctive therapeutic effects of Ag85A/ESAT-6 chimeric DNA, 70 female BALB/c mice (age, 6-8 weeks), were infected intravenously with MDR-TB HB361 [220,000 colony-forming units (CFU)]. The mice were randomly divided into seven groups ( $n=10 /$ group) and were treated as follows: i) Plasmid vector pVAX1 treatment as a negative control; ii) RFP (Shenyang Red Flag Pharmacy Co., Ltd., Shenyang, China) treatment as a negative control; iii) PZA (Chengdu Jinghua Biological Product Co., Ltd., Chengdu, China) treatment as a positive control; iv) Ag85A DNA treatment as a positive control; v) Ag85A/ESAT-6 chimeric DNA treatment; vi) RFP + Ag85A/ESAT-6 chimeric DNA treatment; vii) PZA + Ag85A/ESAT-6 chimeric DNA treatment. Treatment of the mice began on the $2^{\text {nd }}$ day post-infection. RFP $(0.4 \mathrm{mg} / 20 \mathrm{~g})$ and PZA $(0.6 \mathrm{mg} / 20 \mathrm{~g})$ were orally administered every day for 3 months. Ag85A DNA and Ag85A/ESAT-6 chimeric DNA were injected intramuscularly at a dosage of $100 \mu \mathrm{g}$ in $100 \mu \mathrm{l}$ saline five times at 2-week intervals.

In the second experiment, in order to further prove the therapeutic effects of Ag85A/ESAT-6 chimeric DNA, and to evaluate the effects of Ag85A/ESAT-6 chimeric protein enhancement, 90 female BALB/c mice (age, 6-8 weeks) were infected intravenously with M. tuberculosis H37Rv (520,000 CFU). Subsequently, the mice were randomly divided into five groups ( $n=16 /$ group) and were treated as follows: i) Saline treatment as a negative control; ii) plasmid vector pVAX1 treatment as a negative control; iii) Mycobacterium vaccae vaccine (Anhui Longcom Biological Pharmacy Co., Ltd., Anhui, China) treatment as positive control, iv) Ag85A DNA treatment as a positive control; v) Ag85A/ESAT-6 chimeric DNA plus Ag85A/ESAT-6 chimeric protein enhancement treatment. Ag85A DNA and Ag85A/ESAT-6 chimeric DNA were purified using the EndoFree plasmid purification kit (Qiagen) and were prepared by Shanghai H\&G Biotechnology Company (19). Treatment began at the $3^{\text {rd }}$ day post-infection. The Vaccae vaccine $(22.5 \mu \mathrm{g})$ was injected intramuscularly three times at 2-week intervals. Ag85A DNA (100 $\mu \mathrm{g})$ was injected intramuscularly three times at 2-week intervals. Ag85A/ESAT-6 chimeric DNA $(100 \mu \mathrm{g})$ was injected intramuscularly three times at 2-week intervals, and was then boosted once on the $10^{\text {th }}$ day after the final DNA injection by intraperitoneal injection of $90 \mu \mathrm{g} \mathrm{Ag85A/ESAT-6} \mathrm{chimeric} \mathrm{protein.}$

Bacterial counts. In the first experiment, the mice were sacrificed by cervical dislocation under anesthetic with $5 \mathrm{ml}$ dethyl ether (Beijing Chemical Reagents Company, Beijing, China) 3 months post-infection, whereas in the second experiment 10 mice were sacrificed on the $3^{\text {rd }}$ day post-infection, prior to treatment; and 80 mice were sacrificed 7 weeks post-infection. 
Table I. Mortality and time of death of mice with multi-drug-resistant tuberculosis within 3 months of infection with the Mycobacterium tuberculosis clinical isolate HB361.

Time of death

\begin{tabular}{|c|c|c|c|c|}
\hline Group $(n=10)$ & Mortality (no. mice) & Mortality (\%) & (days post-infection) & P-value ${ }^{a}$ \\
\hline Vector & 1 & 10 & 89 & $1.191 \times E-4$ \\
\hline RFP & 1 & 10 & 84 & $1.191 \times E-4$ \\
\hline PZA & 0 & 0 & - & $1.083 \times E-5$ \\
\hline Ag85A DNA & 0 & 0 & - & $1.083 \times E-5$ \\
\hline Ag85A/ESAT-6 chimeric DNA & 10 & 100 & $63-64$ & \\
\hline RFP + Ag85A/ESAT-6 chimeric DNA & 2 & 20 & 91 & $7.145 \times E-4$ \\
\hline PZA + Ag85A/ESAT-6 chimeric DNA & 0 & 0 & - & $1.083 \times E-5$ \\
\hline
\end{tabular}

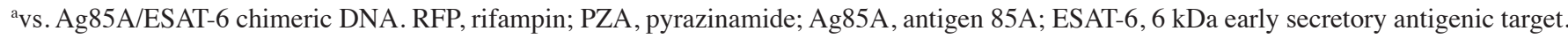

The lungs and spleens of the mice were collected and homogenized in saline. The lungs and spleens from mice in the Ag85A/ESAT-6 DNA group and the lungs from mice in the Ag85A/ESAT-6 chimeric DNA plus Ag85A/ESAT-6 chimeric protein boost group were harvested from mice that had succumbed prior to sacrifice $(n=10)$. The tissue suspension was serially diluted 10 -fold in saline, and $0.1 \mathrm{ml}$ tissue dilutions were plated in duplicate onto Lowenstein-Jensen plates and incubated at $37^{\circ} \mathrm{C}$ for 4 weeks. Colonies on each plate were enumerated and the results were expressed as bacterial load/organ.

Pulmonary histopathological examination. The lungs were fixed in $10 \%(\mathrm{v} / \mathrm{v})$ buffered formalin for $24 \mathrm{~h}$, dehydrated in ethanol and paraffin-embedded. The paraffin-embedded tissue sections $(3 \mu \mathrm{m})$ were prepared and stained with hematoxylin and eosin. Images were obtained using a Huahai Pathological Diagnosis System (Xi'an Huahai Medical Information Technology Co., Ltd., Xi'an, China) and analyzed by a certified pathologist using a light microscope (BX51; Olympus Corporation, Tokyo, China).

Statistical analyses. Data are expressed as the mean \pm standard deviation. Statistical significance between the groups was calculated using one-way analysis of variance followed by Tukey's test, or by Kruskal-Wallis H test for qualitative data. Statistical analyses were performed by SAS version 9.2 (SAS Institute, Cary, NC, USA). $\mathrm{P}<0.05$ was considered to indicate a statistically significant difference.

\section{Results}

Experiment 1. In the first experiment, in which the mice were infected with the MDR clinical isolate HB361, one mouse succumbed in the RFP treatment group 84 days post-infection (mortality 10\%); one mouse succumbed 89 days post-infection in the plasmid vector group (mortality 10\%); two mice succumbed 91 days post-infection in the Ag85A/ESAT-6 DNA + RFP group (mortality 20\%); in the Ag85A/ESAT-6 chimeric DNA group all 10 mice succumbed between 63 and 64 days post-infection (24-48 $\mathrm{h}$ after the $5^{\text {th }}$ Ag85A/ESAT-6 DNA vaccination) (mortality 100\%); whereas all of the mice in
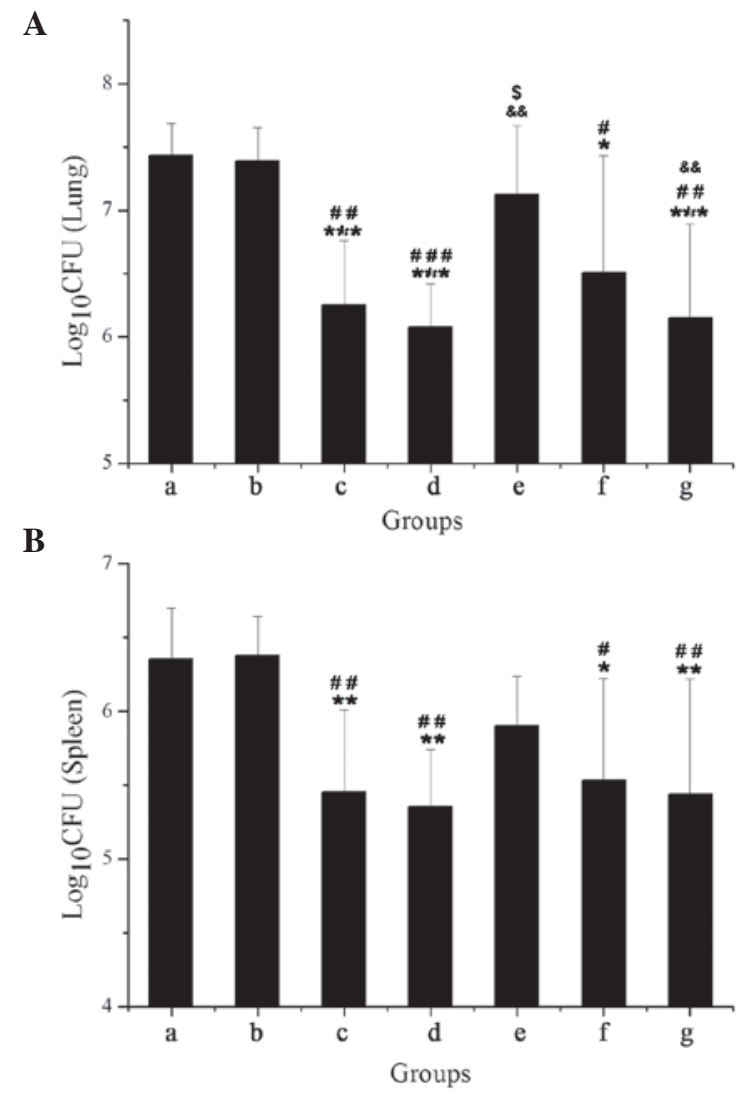

Figure 1. Number of viable bacteria in the (A) lungs and (B) spleens 3 months post-infection with MycobacteriumtuberculosisclinicalisolateHB361.(a)Vector group; (b) rifampin (RFP) group; (c) pyrazinamide (PZA) group; (d) antigen 85A (Ag85A) DNA group; (e) Ag85A/6 kDa early secretory antigenic target(ESAT-6) chimeric DNA group; (f) RFP+Ag85A/ESAT-6 chimeric DNA group; (g) PZA + Ag85A/ESAT-6 chimeric DNA group. The bacterial loads present in the Ag85A/ESAT-6 DNA group were determined from the lungs and spleens of 10 dead mice. Data are presented as the mean \pm standard deviation. ${ }^{*} \mathrm{P}<0.05$, ${ }^{* *} \mathrm{P}<0.01,{ }^{* * *} \mathrm{P}<0.001$ vs. group a; $\mathrm{P}<0.05,{ }^{\# \#} \mathrm{P}<0.01$, ${ }^{\# \#} \mathrm{P}<0.001$ vs. group $\mathrm{b}$; ${ }^{\$} \mathrm{P}<0.05$ vs. group $\mathrm{c} ;{ }^{\& \&} \mathrm{P}<0.01$ vs. group $\mathrm{d}$ and $\mathrm{e}$.

the other treatment groups survived (mortality 0\%) (Table I). Mice infected with the RFP-resistant clinical isolate of $M . t b$ suffered a marked increase in mortality when treated with the DNA vaccine; however, this increase was ameliorated if they were also treated with chemotherapy, not only with PZA (to 

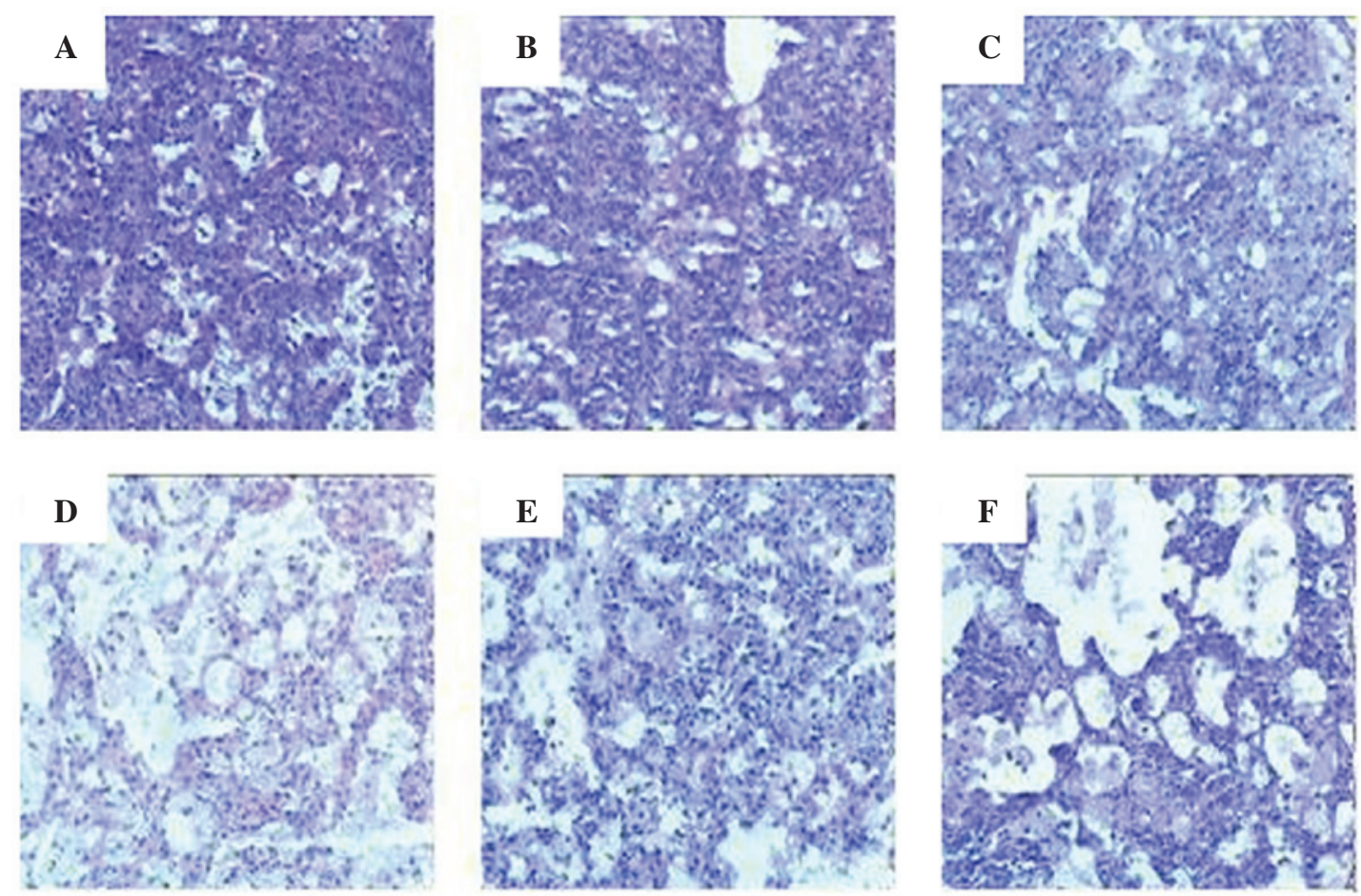

Figure 2. Histopathological pulmonary alterations in a mouse model of multi-drug-resistant tuberculosis. Representative photomicrographs (hematoxylin and eosin; magnification, 200x) of lung tissues obtained from the mice of each group that were still alive 3 months post-infection with Mycobacterium tuberculosis clinical isolate HB361. (A) Vector group; (B) rifampin (RFP) group; (C) pyrazinamide (PZA) group; (D) antigen 85A (Ag85A) DNA group; (E) RFP + Ag85A/6 kDa early secretory antigenic target (ESAT-6) chimeric DNA group; (F) PZA + Ag85A/ESAT-6 chimeric DNA group. Ag85A/ESAT-6 chimeric DNA group is not shown.

which the bacteria were sensitive) but also with RFP (to which the bacteria were clinically defined as resistant).

A total of 3 months post-infection with HB361, substantial bacterial loads were present in the lungs and spleens of the control mice and the mice treated with RFP, as well as in the lungs and spleens of the mice that succumbed following treatment with Ag85A/ESAT-6 chimeric DNA (Fig. 1). Bacterial loads were $\sim 10$-fold lower in the tissues from mice treated with PZA, Ag85A DNA, or Ag85A/ESAT-6 chimeric DNA plus either PZA or RFP.

Microscopic examination of the lung sections from mice in the plasmid vector and RFP-treated groups detected little alteration from the expected histopathological characteristics of murine tuberculosis. Mice in the other groups exhibited more foam cells and multi-nucleated giant cells, but fewer lymphocytes in the lung sections, and the alveolar profiles detected relatively clear and normal structures. The percentage of the total section area that was deemed pathological was 100, 90, 65-75, 30-40, 50-60 and 50-60\% in the plasmid vector group, RFP group, PZA group, Ag85A DNA group, RFP + Ag85A/ESAT-6 chimeric DNA group and PZA + Ag85A/ESAT-6 chimeric DNA group, respectively. Representative histopathological images of each group are presented in Fig. 2.

Experiment 2. In the second experiment, in which mice were infected with $M . t b$ strain H37Rv, three mice succumbed between 12 and 14 days post-infection in the saline group (mortality 19\%); one mouse succumbed 14 days post-infection in the plasmid vector group (mortality 6\%); one mouse succumbed 40 days post-infection in the Vaccae vaccine group (mortality

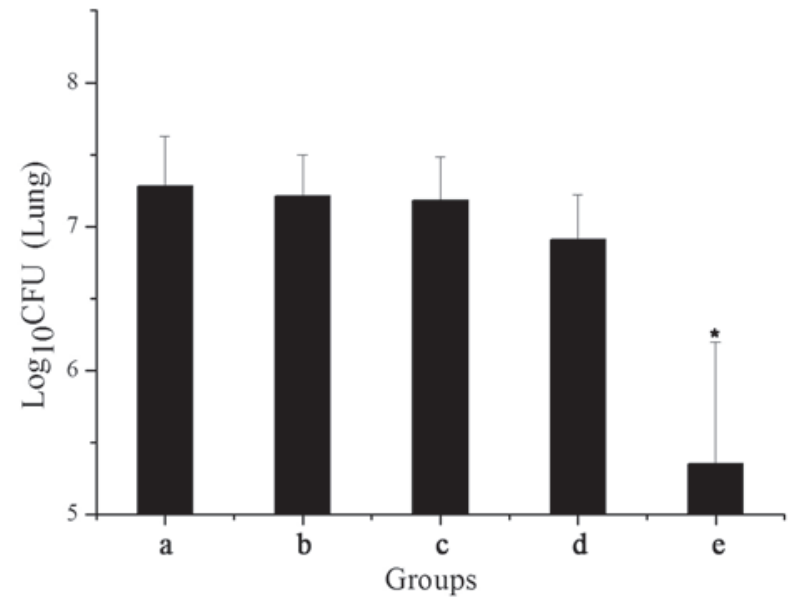

Figure 3. Number of viable bacteria in the lungs at 7 weeks post-infection with Mycobacterium tuberculosis H37Rv. (a) Saline group; (b) vector group; (c) Vaccae vaccine group; (d) Ag85A DNA vaccine group; (e) Ag85A/6 kDa early secretory antigenic target (ESAT-6) chimeric DNA vaccine plus Ag85A/ESAT-6 chimeric protein boost group. Data are presented as the mean \pm standard deviation. ${ }^{*} \mathrm{P}<0.001$ vs. groups a, b, c and d.

6\%); in the Ag85A/ESAT-6 chimeric DNA vaccine plus Ag85A/ESAT-6 chimeric protein boost group, 13 of the 16 mice succumbed 40 days post-infection (48 h after Ag85A/ESAT-6 protein boosting; mortality $81 \%$ ); and all of the mice in the Ag85A DNA group survived (mortality 0\%) (Table II).

The $\log _{10}$ bacterial load in the lungs was determined at 3 days (data not shown) or 7 weeks post-infection (Fig. 3). At 3 days post-infection with $\mathrm{H} 37 \mathrm{Rv}$ (prior to treatment), the $\log _{10}$ 
Table II. Mortality and time of death of mice with tuberculosis within 7 weeks of infection with Mycobaterium tuberculosis H37Rv.

\begin{tabular}{lcccc} 
Group (n=16) & Mortality (no. mice) & Mortality (\%) & $\begin{array}{c}\text { Time of death } \\
\text { (days post-infection) }\end{array}$ & P-value $^{\mathrm{a}}$ \\
\hline Saline & 3 & 19 & $12-14$ & $1.10 \times \mathrm{xE}-3$ \\
Vector & 1 & 6 & 14 & $3.852 \times \mathrm{x}-5$ \\
Vaccae & 1 & 6 & $40^{\mathrm{b}}$ & $3.852 \times \mathrm{x}-5$ \\
Ag85A DNA & 0 & 0 & & $3.224 \times \mathrm{xE}-6$ \\
Ag85A/ESAT-6 chimeric & 13 & 81 & 40 &
\end{tabular}

DNA + Ag85A/ESAT-6 chimeric protein boost

${ }^{\mathrm{a}} \mathrm{vs}$. Ag85A/ESAT-6 chimeric DNA + Ag85A/ESAT-6 chimeric protein boost. ${ }^{\mathrm{b}}$ After third immunization. Ag85A, antigen 85A; ESAT-6, 6 kDa early secretory antigenic target.
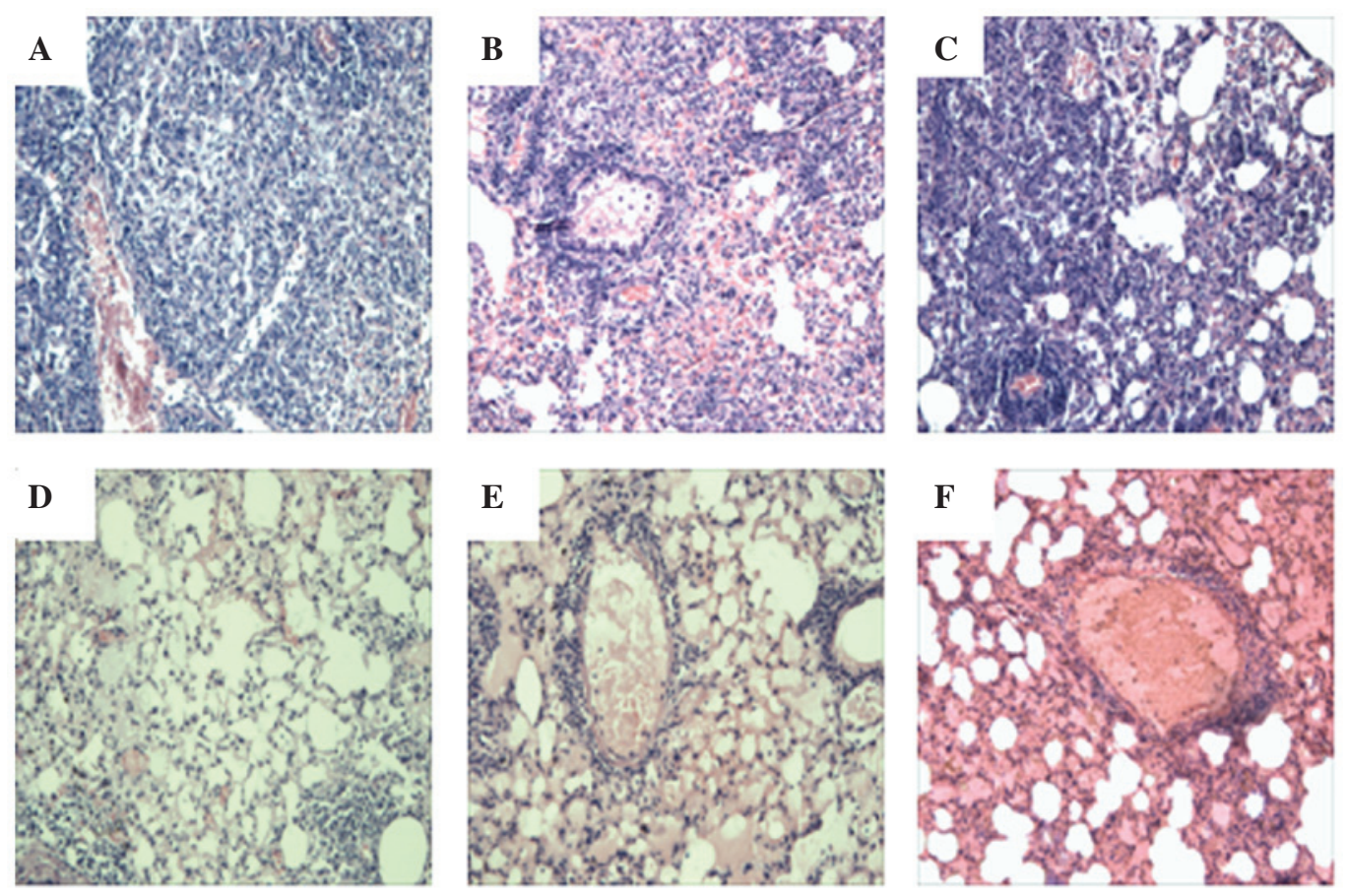

Figure 4. Histopathological pulmonary alterations in a mouse model of drug-sensitive tuberculosis. Representative photomicrographs (hematoxylin and eosin; magnification, 100x) of lung tissue obtained from 16 mice (both alive and dead) in each group 7 weeks post-infection with Mycobacterium tuberculosis $\mathrm{H} 37 \mathrm{Rv}$ are shown. (A) Saline group; (B) vector group; (C) Vaccae vaccine group; (D) antigen 85A (Ag85A) DNA vaccine group; (E) alive and (F) dead mice from the Ag85A/6 kDa early secretory antigenic target (ESAT-6) chimeric DNA vaccine plus Ag85A/ESAT-6 chimeric protein boost group.

bacterial load in the lungs from 10 mice was 7.04. At 7 weeks post-infection, compared with the CFU in the control groups treated with saline (7.31) or empty DNA vaccine vector (7.20), treatment with Vaccae had no effect on CFU (7.21). The load was slightly but significantly lower $(6.93 ; \mathrm{P}<0.05)$ following treatment with Ag85A DNA, and was almost $2 \log _{10}$ lower in the mice that succumbed following treatment with Ag85A/ESAT-6 chimeric DNA plus Ag85A/ESAT-6 chimeric protein boost $\left(5.37 \log _{10} ; \mathrm{P}<0.001\right)$.

Histopathological examination of the lungs demonstrated that in general the vaccinated groups exhibited reduced pathology compared with in the control groups receiving saline or plasmid vector (Fig. 4). At 3 days post-infection, a small scope of consolidation in the local area, scattered small lung lesions, transudate, and hyperaemia in the alveoli were observed (data not shown). At 7 weeks post-infection, more lymphocytes, extensive lung lesions, hyperaemia in the alveoli and damaged structures were observed in the lung sections of the mice in the saline and vector groups. In the Vaccae vaccine group lung lesions were limited, and moderate lymphocyte infiltration and relatively clear and normal structures were observed. In the Ag85A DNA group, lesions were slight with few lymphocytes, and the alveoli exhibited relatively clear and normal structures. Mice in the Ag85A/ESAT-6 chimeric DNA plus Ag85A/ESAT-6 chimeric protein boost group (both alive and dead) all exhibited reduced lesions; however, more transudatory proteins and hyperaemia were detected in the alveoli, and dilated 
capillary vessels and more lymphocytes surrounding the capillary vessels were observed.

\section{Discussion}

The present study determined whether immunotherapy with an Ag85A/ESAT-6 chimeric DNA vaccine combined with conventional chemotherapy was more effective for the treatment of TB or MDR-TB in mice, compared with treatment with a single Ag85A DNA vaccine.

The immunotherapeutic effects of DNA vaccines expressing Ag85A have been reported in murine TB (5-7), and have been shown to exert action against MDR-TB (20). In addition, ESAT-6 DNA vaccines have been reported to have immunotherapeutic and immune protective effects in mice infected with TB $(11,12,22,23)$. However, the present study did not detect an enhanced protective effect of a DNA vaccine expressing the fusion protein. It was confirmed that repeated dosing with Ag85A DNA exerted therapeutic effects against H37Rv infection, reducing both CFU and pathology. Conversely, the disease worsened following vaccination with Ag85A/ESAT-6 DNA in mice infected with either H37Rv or HB361. Repeated dosing with the plasmid accelerated mortality without having a marked effect on HB361 CFU; however, some granuloma formation was replaced with vasculitis and edema (experiment 1). Similarly, boosting immunity using the fusion protein following Ag85A/ESAT-6 DNA vaccination (experiment 2) accelerated the lethal outcome of infection with H37Rv, thus suggesting that the phenomenon was protein-driven and was not dependent upon the strain of $M$. $t b$. In addition, granulomas were not a prominent feature of the histopathology; however, vasculitis, hyperaemia and edema appeared likely to contribute toward mortality. Reduced bacterial load was detected in the dead mice, thus suggesting that there may have been a bacterial killing component to the immune response, however this was not investigated.

The harmful effect of the vaccine in HB361-infected mice was much reduced when the vaccine was given as an adjunct to treatment with PZA, a chemotherapeutic agent to which the bacterium was susceptible. This is consistent with an immunopathology that depends upon antigen load, which was reduced by the drug. Notably, however, the harmful effects were also slightly reduced when the vaccine was administered alongside RFP; mortality, pathology and CFU were all reduced, even though the bacteria were clinically defined as resistant to RFP and monotherapy with RFP was ineffective. Whether this finding was due to a direct modulation of the immune response by RFP, as observed elsewhere $(24,25)$, or due to a greater susceptibility of the bacteria to the drug in vivo, as implied by other studies (26) is currently unknown.

The present study hypothesized that the harmful effects of DNA expressing the chimeric Ag85A/ESAT-6 antigen in TB-infected mice may have been a consequence of the development of hypersensitivity. Histopathological analysis detected slight lesions, increased transudate and hyperaemia in alveoli, dilated capillary vessels, and increased lymphocytes surrounding the capillary vessels in both experiments. This was the case whether the mice had naturally succumbed or were sacrificed following Ag85A/ESAT-6 treatment.
Previous studies, which used the ESAT-6 DNA vaccine or Ag85B/ESAT-6 fusion protein vaccine (as an adjunct to IC31) as preventative vaccines in mice or humans, or as therapeutic vaccines in mice $(11,12,16,20,27)$, did not detect any adverse reaction. However, in the present study, inserting two esat-6 genes within the ag85a gene created a novel entity capable of converting a partially protective immune response into a harmful immune response. The underlying mechanisms have not yet been explored, but may reflect the increased expression levels of ESAT-6 relative to Ag85A. ESAT-6 is not a biologically inert protein antigen, and has been proven to act as a virulence factor. ESAT-6 can cause lysis of red blood cells and macrophages by membrane pore formation (28). In addition, it can cause disruption of membrane conductance, destruction of artificial planar bilayers (28), formation of membrane pores, and induction of apoptosis in macrophages by binding to laminin and activating caspase expression $(29,30)$. Therefore, ESAT-6 may act as a cytolytic pore-forming toxin, inducing lysis of red blood cells, macrophages and lung epithelial cells, and this may be considered the primary mechanism underlying the hypersensitivity reaction detected in the present study.

In conclusion, in the context of therapeutic application against TB in mice, relatively high expression of ESAT-6 antigen via multiple immunizations with Ag85A/ESAT-6 chimeric DNA induced a hypersensitivity reaction and accelerated mortality of the mice. These results suggested that ESAT-6 may not be suitable for use in immunotherapeutic vaccines against TB.

\section{Acknowledgements}

The present study was supported by grants from the Serious Infectious Diseases Special Foundation of China (grant nos. 2008ZX10003013-2 and 2012ZX10003008002), the WHO IVR Steering Committee (grant no. V25-181-202) and the National Nature and Science Foundation of China (grant no. 30070730). The study has been presented as part of a poster at the 3rd International Conference on Vaccines and Vaccination.

\section{References}

1. Glaziou P, Falzon D, Floyd K and Raviglione M: Global epidemiology of tuberculosis. Semin Respir Crit Care Med 34: 3-16, 2013.

2. WHO/IUATLD Global Project on Anti-Tuberculosis Drug Resistance Surveillance. Anti-tuberculosis drug resistance in the world: Third global report/the WHO/IUATLD Global Project on Anti-Tuberculosis Drug Resistance Surveillance, 1999-2002.

3. Mori T: MDR-TB - its characteristics and control in Asia-Pacific rim symposium in USJCMSP 10th international conference on emerging infectious diseases in the Pacific rim. Tuberculosis (Edinb) 87 (Suppl 1): S5-S9, 2007.

4. The Office of the Fifth National TB Epidemiological Survey, Technical Guidance Group of the Fifth National TB Epidemiological Survey: The Fifth National Tuberculosis Epidemiological Survey in 2010. Chin J Antitubere 34: 485-508, 2010, 2012.

5. Ha SJ, Jeon BY, Youn JI, Kim SC, Cho SN and Sung YC: Protective effect of DNA vaccine during chemotherapy on reactivation and reinfection of Mycobacterium tuberculosis. Gene Ther 12: 634-638, 2005.

6. Silva CL, Bonato VL, Coelho-Castelo AA, De Souza AO, Santos SA, Lima KM, Faccioli LH and Rodrigues JM: Immunotherapy with plasmid DNA encoding mycobacterial hsp65 in association with chemotherapy is a more rapid and efficient form of treatment for tuberculosis in mice. Gene Ther 12: 281-287, 2005. 
7. Ha SJ, Jeon BY, Kim SC, Kim DJ, Song MK, Sung YC and Cho SN: Therapeutic effect of DNA vaccines combined with chemotherapy in a latent infection model after aerosol infection of mice with Mycobacterium tuberculosis. Gene Ther 10: 1592-1599, 2003.

8. Zhu D, Jiang S and Luo X: Therapeutic effects of Ag85B and MPT64 DNA vaccines in a murine model of Mycobacterium tuberculosis. Vaccine 23: 4619-4624, 2005.

9. Cooper AM, Dalton DK, Stewart TA, Griffin JP, Russell DG and Orme IM: Disseminated tuberculosis in interferon gamma gene-disrupted mice. J Exp Med 178: 2243-2247, 1993.

10. Flynn JL, Chan J, Triebold KJ, Dalton DK, Stewart TA and Bloom BR: An essential role for interferon gamma in resistance to Mycobacterium tuberculosis infection. J Exp Med 178: 2249-2254, 1993.

11. Lowrie DB, Tascon RE, Bonato VL, Lima VM, Faccioli LH, Stavropoulos E, Colston MJ, Hewinson RG, Moelling K and Silva CL: Therapy of tuberculosis in mice by DNA vaccination. Nature 400: 269-271, 1999

12. Fan X, Gao Q and Fu R: DNA vaccine encoding ESAT-6 enhances the protective efficacy of BCG against Mycobacterium tuberculosis infection in mice. Scand J Immunol 66: 523-528, 2007.

13. Harboe M, Oettinger T, Wiker HG, Rosenkrands I and Andersen P: Evidence for occurrence of the ESAT-6 protein in Mycobacterium tuberculosis and virulent Mycobacterium bovis and for its absence in Mycobacterium bovis BCG. Infect Immun 64: 16-22, 1996.

14. Brandt L, Oettinger T, Holm A, Andersen AB and Andersen P. Key epitopes on the ESAT- 6 antigen recognized in mice during the recall of protective immunity to Mycobacterium tuberculosis. J Immunol 157: 3527-3533, 1996.

15. Andersen P and Heron I: Specificity of a protective memory immune response against Mycobacterium tuberculosis. Infect Immun 61: 844-851, 1993.

16. Langermans JA, Doherty TM, Vervenne RA, van der Laan T, Lyashchenko K, Greenwald R, Agger EM, Aagaard C, Weiler H, van Soolingen D, et al: Protection of macaques against Mycobacterium tuberculosis infection by a subunit vaccine based on a fusion protein of antigen $85 \mathrm{~B}$ and ESAT-6. Vaccine 23 2740-2750, 2005.

17. Pathan AA, Minassian AM, Sander CR, Rowland R, Porter DW, Poulton ID, Hill AV, Fletcher HA and McShane H: Effect of vaccine dose on the safety and immunogenicity of a candidate $\mathrm{TB}$ vaccine, MVA85A, in BCG vaccinated UK adults. Vaccine 30 5616-5624, 2012

18. Bryder K, Sbai H, Nielsen HV, Corbet S, Nielsen C, Whalen RG and Fomsgaard A: Improved immunogenicity of HIV-1 epitopes in HBsAg chimeric DNA vaccine plasmids by structural mutations of HbsAg. DNA Cell Biol 18: 219-225, 1999.

19. Li Z, Song D, Zhang H, He W, Fan X, Zhang Y, Huang J, Wang X, Liu $Q$ and Xiong S: Improved humoral immunity against tuberculosis ESAT-6 antigen by chimeric DNA prime and protein boost strategy. DNA Cell Biol 25: 25-30, 2006.
20. Liang Y, Wu X, Zhang J, Li N, Yu Q, Yang Y, Bai X, Liu C, Shi Y, Liu Q, et al: The treatment of mice infected with multi-drug-resistant Mycobacterium tuberculosis using DNA vaccines or in combination with rifampin. Vaccine 26: 4536-4540, 2008.

21. Chinese Antituberculosis Association: Chinese laboratory science procedure of diagnostic bacteriology in tuberculosis. Wang SM (ed.). China Education Culture Press, Beijing, pp46-61, 2006.

22. Skjøt RL, Oettinger T, Rosenkrands I, Ravn P, Brock I, Jacobsen S and Andersen P: Comparative evaluation of low-molecular-mass proteins from Mycobacterium tuberculosis identifies members of the ESAT- 6 family as immunodominant T-cell antigens. Infect Immun 68: 214-220, 2000.

23. Wang QM, Sun SH, Hu ZL, Yin M, Xiao CJ and Zhang JC: Improved immunogenicity of a tuberculosis DNA vaccine encoding ESAT6 by DNA priming and protein boosting. Vaccine 22: 3622-3627, 2004

24. Humber DP, Nsanzumuhire H, Aluoch JA, Webster AD, Aber VR, Mitchison DA, Girling DJ and Nunn AJ: Controlled double-blind study of the effect of rifampin on humoral and cellular immune responses in patients with pulmonary tuberculosis and tuberculosis contacts. Am Rev Respir Dis 122: 425-436, 1980.

25. Ziglam HM, Daniels I and Finch RG: Immunomodulating activity of rifampicin. J Chemother 16: 357-361, 2004.

26. Chanwong S, Maneekarn N, Makonkawkeyoon L and Makonkawkeyoon S: Intracellular growth and drug susceptibility of Mycobacterium tuberculosis in macrophages. Tuberculosis 87: 130-133, 2007.

27. van Dissel JT, Arend SM, Prins C, Bang P, Tingskov PN, Lingnau K, Nouta J, Klein MR, Rosenkrands I, Ottenhoff TH, et al: Ag85B-ESAT-6 adjuvanted with IC31 promotes strong and long-lived Mycobacterium tuberculosis specific T cell responses in naïve human volunteers. Vaccine 28: 3571-3581, 2010.

28. Smith J, Manoranjan J, Pan M, Bohsali A, Xu J, Liu J, McDonald KL, Szyk A, LaRonde-LeBlanc N and Gao LY: Evidence for pore formation in host cell membranes by ESX-1-secreted ESAT-6 and its role in Mycobacterium marinum escape from the vacuole. Infect Immun 76: 5478-5487, 2008.

29. Derrick SC and Morris SL: The ESAT6 protein of Mycobacterium tuberculosis induces apoptosis of macrophages by activating caspase expression. Cell Microbiol 9: 1547-1555, 2007.

30. van der Wel N, Hava D, Houben D, Fluitsma D, van Zon M, Pierson J, Brenner $\mathrm{M}$ and Peters PJ: M. tuberculosis and $M$. leprae translocate from the phagolysosome to the cytosol in myeloid cells. Cell 129: 1287-1298, 2007. 